\title{
Parameterized Dynamic Variants of Red-Blue Dominating Set
}

\author{
Faisal N. Abu-Khzam ${ }^{1}\left(\mathbb{D}\right.$, Cristina Bazgan ${ }^{2}\left(\mathbb{D}\right.$, and Henning Fernau $^{3(\otimes)}(\mathbb{D}$ \\ 1 Department of Computer Science and Mathematics, Lebanese American University, \\ Beirut, Lebanon \\ faisal.abukhzam@lau.edu.lb \\ 2 Université Paris-Dauphine, PSL University, CNRS, LAMSADE, \\ 75016 Paris, France \\ cristina.bazgan@lamsade.dauphine.fr \\ 3 Universität Trier, \\ Fachber. 4 - Abteilung Informatikwissenschaften, 54286 Trier, Germany \\ fernau@uni-trier.de
}

\begin{abstract}
We introduce a parameterized dynamic version of the RedBlue Dominating Set problem and its partial version. We prove the fixedparameter tractability of the dynamic versions with respect to the (so called) edit-parameter while they remain $\mathcal{W}[2]$-hard with respect to the increment-parameter. We provide a complete study of the complexity of the problem with respect to combinations of the various parameters.
\end{abstract}

Keywords: Dynamic problems $\cdot$ Reoptimization $\cdot$ Parameterized complexity $\cdot$ Set cover $\cdot$ Hitting set

\section{Introduction}

In the Red-Blue Dominating Set problem (henceforth $R B D S$ ), we are given a graph $G=(R \cup B, E)$ such that $R \cap B=\emptyset$, together with an integer $s \geq 0$, and we are asked whether $R$ contains a subset $S$ of cardinality at most $s$ such that every element of $B$ has at least one neighbor in $S$. In this case the elements of $R$ and $B$ are called red and blue vertices respectively, and $S$ is a red-blue dominating set of $G$. We shall also refer to $G$ as a red-blue graph. It is wellknown that $R B D S$ is equivalent to Set Cover as well as to Hitting Set. ${ }^{1}$

In this paper we are interested in the parameterized complexity of dynamic versions of $R B D S$ and some of its variants. In such dynamic settings, originally defined by Downey et al. in [12] in the context of the Dominating Set problem, we assume the edges of the input graph $G$ can appear or disappear with time, so an initially feasible $R B D S$ solution $S$ (not necessarily optimal) may no longer

\footnotetext{
${ }^{1}$ The problem should not be confused with Red-Blue Set Cover as studied in [7].
}

This work was supported by the bilateral research cooperation CEDRE between France and Lebanon (grant number 40334SG).

(C) Springer Nature Switzerland AG 2020

A. Chatzigeorgiou et al. (Eds.): SOFSEM 2020, LNCS 12011, pp. 236-247, 2020.

https://doi.org/10.1007/978-3-030-38919-2_20 
dominate all of $B$ and we want to construct another solution $S^{\prime}$ so that the Hamming distance between $S$ and $S^{\prime}$ is minimized. The problem is formally defined as follows.

\section{Dynamic Red-Blue Dominating Set $(D R B D S)$}

Given: Two red-blue graphs $G=(R \cup B, E)$ and $G^{\prime}=\left(R \cup B, E^{\prime}\right)$, a subset $S$ of $R$ that is a red-blue dominating set of $G$, integers $k$ and $r$ such that $d_{H}\left(E, E^{\prime}\right) \leq k$. Question: Is there a subset $S^{\prime}$ of $R$ such that $d_{H}\left(S, S^{\prime}\right) \leq r$ and $S^{\prime}$ is a red-blue dominating set of $G^{\prime}$ ?

By analogy to operations on characteristic vectors, the Hamming distance between two sets (subsets of the same, mostly implicitly given ground set) refers to the cardinality of the symmetric set difference, i.e., $d_{H}\left(S, S^{\prime}\right)=\left|S \Delta S^{\prime}\right|$.

Remark 1. Since $r$ is a bound on the Hamming distance between $S$ and $S^{\prime}$, we are not necessarily interested in deleting elements from $S$. Therefore we can always assume $S \subseteq S^{\prime}$ according to the formulation above. Then, $d_{H}\left(S, S^{\prime}\right)=\left|S^{\prime}\right|-|S|$. Hence, $r$ is called the increment-parameter. Similarly, in view of the problem at hand, it suffices to consider the special case when $E^{\prime} \subseteq E$, i.e., $E^{\prime}$ resulted from $E$ by removing some edges. Then, $d_{H}\left(E, E^{\prime}\right)=|E|-\left|E^{\prime}\right|$. More precisely, if $G=(R \cup B, E), G^{\prime}=\left(R \cup B, E^{\prime}\right), S, k$ and $r$ form an instance of DRBDS, then also $\hat{G}=\left(R \cup B, E \cup\left(E^{\prime} \backslash E\right)\right), G^{\prime}=\left(R \cup B, E^{\prime}\right), S, k$ and $r$ form an instance of $D R B D S$ as well, and $S^{\prime} \subseteq R$ is (or is not) a red-blue dominating set of $G^{\prime}$ in both cases. We call $k$ the edit-parameter.

Notice that $D R B D S$ could be also viewed as a reoptimization variant of Set Cover as defined in [3]. However, the more interesting approximation results obtained in that paper rely on the given solution to be optimum, a condition that is relaxed in this paper. So, we are following here the terminology introduced in $[1,12]$, which is not the same as later used in [2] under a similar name.

\section{Motivation}

In social networks, there is a growing interest in domination problems that can model the search for influencers in the best way, in particular aiming at people who can spread positive influence and participate in launching a global campaign such as, for example, a non-smoking campaign. In such a context, the network consists of two types of people: the set $R$ of non-smokers (who are not to be convinced but can help in convincing others) and the set $B$ which consists of those who are known to have the smoking habit. The natural social network of friendship relations can be modeled as a graph.

Ideally one would seek a set of individuals $S \subseteq R$ (to serve as influencers) that are friends of all the elements of $B$. However, in real settings, the objective would be to affect (or, in a more formal sense, to dominate) as many elements of $B$ as possible. This latter objective is modeled using the partial version of $R B D S$, originally defined in [15] in the context of learning theory. Moreover, it would be more realistic to assume someone is influenced by the non-smoking campaign if there are at least a number, say $q$, of his or her friends who serve as 
influencers. This gives rise to the $q-R B D S$ problem which differs from $R B D S$ only in the domination condition requiring each element of $B$ to have at least $q$ neighbors in $S \subseteq R .^{2}$

We shall study the partial $q-R B D S$ problem, among other variants, and focus on its parameterized dynamic version to cope with settings where the network is changing with time.

Another interesting scenario where dynamic $R B D S$ could be useful is when the red and blue vertices correspond to stores and customers, respectively, and the links are based on credit card transactions: an edge $u v$ means customer $u$ frequently purchases items from store $v$. In such a setting, it would be interesting to have a smallest possible list of stores that are preferred by a large number (or majority) of customers. Moreover, since data from credit card transactions is dynamic, and the interests of customers change with time, some links in the corresponding red-blue graph can appear or disappear, which might require updating a previously computed (partial) $R B D S$ solution. The so-called partial $D R B D S$ problem is obviously the right model in this case. In fact, the more general partial (dynamic) $q-R B D S$ problem could be of interest, as well. This is another direction studied here.

Finally, let us mention another motivation for dynamic problems as considered in the paper: assume we have found (over time, with experience) a nice solution to the problem that we are interested in. Here, nice does not necessarily mean smallest or largest, but just satisfying a number of properties, out of which some are formalized and are those we wish to keep up even if the situation changes slightly. As the previous solution was nice, we do not want to change it too much. This justifies the general assumption (important in the context of Parameterized Complexity) that the two change parameters can be assumed to be small.

Throughout this paper, we adopt common graph-theoretic terminology and notations. Apart from the problems mentioned above, we will discuss quite a number of auxiliary problems that might be of independent interest. The paper is structured as follows. In the next section, we study the complexity of the dynamic version of the Red-Blue Dominating Set problem. The partial version is studied in Sect. 3, while the last section briefly addresses approximability but focuses on open problems.

\section{Complexity of Dynamic Red Blue Dominating Set}

The fact that $D R B D S$ is $\mathcal{N} \mathcal{P}$-hard is obvious. It follows immediately from the $\mathcal{N} \mathcal{P}$-hardness of $R B D S$ itself. To see this, let $(G=(R \cup B, E), s)$ be an instance of $R B D S$; construct two graphs $G_{1}$ and $G_{2}$ as follows: ${ }^{3} G_{1}$ is obtained from $G$ by adding a special vertex $w$ and joining it by $|B|$ edges to each vertex of $B$.

\footnotetext{
${ }^{2}$ In this model, possibly also the influence of smokers on their smoking friends should be taken into account. This would lead to notions like alliances or monopolies as discussed in [13]. We are not going into this direction in this paper.

${ }^{3}$ We also refer to the general discussions of hardness for dynamic problems in [5].
} 
$G_{2}$ is obtained by deleting the $k=|B|$ edges incident on $w$. Now set $S=\{w\}$ and $r=s$ to obtain the dynamic $R B D S$ instance. Obviously, any solution $S^{\prime}$ is equivalent to a solution to the given $R B D S$ instance and vice versa.

We now define the Need-Based Red-Blue Dominating Set problem and use it to obtain some algorithmic results which might be of independent interest. ${ }^{4}$ Need-Based Red-Blue Dominating Set (NB-RBDS)

Given: A red-blue graph $G=(R \cup B, E)$ together with integers $s, q \geq 0$ and a function $\eta: B \longrightarrow\{0,1, \ldots, q\}$.

Question: Does $R$ contain a subset $D$ of cardinality at most $s$ such that every element $b$ of $B$ has at least $\eta(b)$ neighbors in $D$ ?

Notice that in approximation algorithms, this type of problems has been studied as a special case of covering integer programs, see [22] as an example reference. Namely, considering $\boldsymbol{\eta}$ as a $|B|$-dimensional vector and thinking of $\boldsymbol{x}$ as a $|R|$-dimensional binary solution vector, as well as $A$ as the $|B| \times|R|$-biadjacency matrix of the bipartite graph $G$, then $N B-R B D S$ asks to find a solution vector $\boldsymbol{x}$ with at most $s$ one-entries that satisfies $A \boldsymbol{x} \geq \boldsymbol{\eta}$. This translation immediately provides an $O\left(|R|^{O(|R|)}\right)$-algorithm based on Lenstra's results [18]. We present an alternative approach now, which is better roughly in the case when $(q+1)^{|B|}<$ $|R|^{|R|}$.

Notice that vertices with $\eta(v)=0$ are trivially satisfied, so they can be removed from the instance. Hence, we can (tacitly) assume $\eta: B \longrightarrow\{1, \ldots, q\}$.

Theorem 1. NB-RBDS is fixed-parameter tractable with respect to $|B|$ and $q$ as parameters.

Proof. Let $R=\left\{r_{1}, \ldots, r_{n}\right\}, B=\left\{b_{1}, \ldots, b_{m}\right\}$ and $q=\max _{1 \leq i \leq m}\left\{\eta\left(b_{i}\right)\right\}$. We show how to construct a solution $S$ in time $O\left((q+1)^{|B|}\right)$ by using dynamic programming.

Consider the set of all functions from $B$ to the set $\{0,1, \ldots, q\}$. There are at most $(q+1)^{|B|}$ many of them. A more precise upper-bound on their number is $\prod_{b \in B}(\eta(b)+1)$, as each such function can be represented by a vector $\left(x_{1}, \ldots, x_{m}\right)$, where $x_{i} \in\left\{0,1, \ldots, \eta\left(b_{i}\right)\right\}$ and $m=|B|$.

In the dynamic programming algorithm that we describe next, our target vector is $\boldsymbol{x}=\left(x_{1}, \ldots, x_{m}\right)$, where $x_{i}$ is the number of elements of $R$ that are still needed to (finally) cover $b_{i}$ with $\eta\left(b_{i}\right)$ elements of $R$. Let $R_{j}=\left\{r_{1}, r_{2}, \ldots, r_{j}\right\}$ and let $C[\boldsymbol{x}, j]$ be the minimum number of elements of $R_{j}$ needed to be added to $S$ in order to cover each $b_{i}$ with $x_{i}$ elements from $R_{j}$. Hence, we initialize $C[\mathbf{0}, 0]=0$ and $C[\boldsymbol{x}, 0]=\infty$ for all $\boldsymbol{x} \neq \mathbf{0}$. Then we have the following recursion for $j>0$ :

$$
C[\boldsymbol{x}, j]=\min \left\{C[\boldsymbol{x}, j-1], 1+C\left[\max \left\{\boldsymbol{x}-\chi_{N\left(r_{j}\right)}, \mathbf{0}\right\}, j-1\right]\right\},
$$

\footnotetext{
${ }^{4}$ The notion of capacitated domination is related. Unfortunately, this notion is not used consistently in the literature. While in [14], both capacities and demands are associated to vertices, so that capacities equal to degrees and demands equal to needs would be exactly a need-based variant of domination, in $[6,11]$, there is no demand function. However, we are not going into this direction here, also because for our purposes, the need-based variation is rather an auxiliary problem.
} 
where $\chi_{N\left(r_{j}\right)}$ is the characteristic vector of $N\left(r_{j}\right) \subseteq B$; the maximum operation is understood component-wisely. So in the case where $r_{j}$ is in a smallest solution from $R_{j}$ we subtract 1 from each $x_{i}$ where $b_{i} \in N\left(r_{j}\right)$. Obviously, the bottom up dynamic programming approach would compute any target vector in time $O\left((q+1)^{m}|R|\right)$. This could be speed up by not considering target vectors with components $x_{i}>\eta\left(b_{i}\right)$.

Observe that one could view our problem as a (very) special case of the Hitting Set of Bundles problem considered in [10]. In fact, for each vertex $b \in B$, we would introduce the set system $S(b)$ of all $\eta(b)$-element subsets of $N(b)$ and the question would be to select a subset $s(b) \in S(b)$ for each $b \in B$ so that the set $\bigcup_{b \in B} s(b) \subseteq R$ has cardinality at most $s$. Unfortunately, we arrive at a very special case of Hitting Set of Bundles, so that the parameterized complexity results known for that problem are not very helpful in our case. In particular, the situation where Damaschke could prove $\mathcal{W}[1]$-hardness, when the bundles have size at most two, we would face the situation when $q=2$ is a constant and we parameterize by $|B|$ and $s$, a situation covered (in a much stronger sense) by the previous theorem, leading to an $\mathcal{F} \mathcal{P} \mathcal{T}$ result in our scenario.

Note that the solution size, $s$, was not treated as a parameter in the proof above, so the dynamic programming algorithm finds a solution of minimum size in $R$. We can (therefore) obtain the following result. Notice that we now fix $q \geq 1$ as part of the problem definition.

Corollary 1. Dynamic $q-R B D S$ is fixed-parameter tractable with respect to the edit-parameter $k$.

Proof. Consider two red-blue graphs $G=(R \cup B, E)$ and $G^{\prime}=\left(R \cup B, E^{\prime}\right)$, a subset $S$ of $R$ that is a red-blue dominating set of $G$, integers $k$ and $r$ such that $d_{H}\left(E, E^{\prime}\right) \leq k$. By Remark 1 , we can assume that $E^{\prime} \subseteq E$. Let $B^{\prime} \subseteq B$ be the set of elements of $B$ that have less than $q$ neighbors in $S$ after the deletion of at most $k$ edges when moving from $E$ to $E^{\prime}$. Let $B^{\prime}=\left\{b_{1}, b_{2}, \ldots, b_{k^{\prime}}\right\}, k^{\prime} \leq k$ (since at most $k$ edges are deleted). Let $\eta\left(b_{i}\right)=\max \left\{q-\left|N_{G^{\prime}}\left(b_{i}\right) \cap S\right|, 0\right\}$. In other words, $\eta\left(b_{i}\right)$ is the number of elements of $R$ that are still needed to dominate $b_{i}$ with $q$ (red) neighbors. Let $q^{\prime}=\max _{1 \leq i \leq k^{\prime}}\left\{\eta\left(b_{i}\right)\right\}$. Obviously $q^{\prime} \leq k$.

Now we are left with the instance $\left(G=\left(R \cup B^{\prime}, E\right), r, \eta\right)$ of the $N B-R B D S$ problem, which is solvable in $O\left(\left(q^{\prime}+1\right)^{\left|B^{\prime}\right|}\right)$. This proves our assertion, knowing that both $q^{\prime}$ and $\left|B^{\prime}\right|$ are bounded above by $k$.

The following corollary follows immediately from the above; it corresponds to the case $q=1$.

Corollary 2. Dynamic RBDS is fixed-parameter tractable with respect to the edit-parameter $k$.

Remark 2. More precisely, the proof of Corollary 1 shows that we can estimate the running time as $O^{*}\left(2^{k}\right)$, as $\eta: B^{\prime} \rightarrow\{0,1\}$. Notice that algorithms of the form $O^{*}\left(2^{o(k)}\right)$ are not to be expected under the Set Cover Conjecture, see [9], because it is possible to formulate Set Cover as Dynamic $R B D S$. In essence, this is also done in the proof of the next theorem, in an even more general setting. 
Now we turn our attention to the increment-parameter, $r$. It was shown in $[1,12]$ that Dynamic Dominating Set is $\mathcal{W}[2]$-hard when parameterized by the increment-parameter $r$ only. We show the same for $q-R B D S$.

Theorem 2. For any $q \geq 1$, Dynamic $q-R B D S$ is $\mathcal{W}[2]$-hard with respect to the increment-parameter $r$.

Proof. By reduction from the $\mathcal{W}[2]$-hard $R B D S$ problem. Let $(G=(R \cup B, E), r)$ be an instance of $R B D S$. We construct an instance $\left(G_{1}, G_{2}, S, k, r\right)$ of Dynamic $q-R B D S$ as follows.

$G_{1}$ is obtained from $G$ by adding $q$ red vertices forming the set $S$ that, together with $B$, induces a complete bipartite subgraph. Let $S=\left\{w_{1}, w_{2} \ldots, w_{q}\right\}$. Then $G_{1}=\left(R^{\prime} \cup B, E_{1}\right)$ where $R^{\prime}=R \cup S$ and $E_{1}=E \cup\left\{v w_{i}: v \in B, 1 \leq i \leq q\right\}$. Let $G_{2}=\left(R^{\prime} \cup B, E_{2}\right)$ where $E_{2}=E_{1} \backslash\left\{v w_{1}: v \in B\right\}$ and $k=|B|$. In other words, every element of $B$ is dominated in $G_{1}$ by the $q$ vertices of $S$. However, in $G_{2}$ every element of $B$ is dominated by the $q-1$ vertices of $S \backslash\left\{w_{1}\right\}$. A solution $S^{\prime}$ of this Dynamic $q-R B D S$ instance must contain at most $r$ vertices from $R$ that dominate $B$.

Remark 3. It is not very difficult to design a multi-tape Turing machine that solves Dynamic $q-R B D S$ by first guessing the (at most) $r$ vertices to be added to the existing red-blue dominating set and then verifying this guess by using one tape per vertex (better said neighborhood) in the spirit of [8]. The only difference to the classical approach is that some head positions have to be individually set by the reduction machine that constructs this Turing machine, based on the information how the given set $S$ already dominates other vertices.

Corollary 3. Dynamic RBDS is $\mathcal{W}[2]$-complete with increment-parameter $r$.

\section{The Partial Dynamic RBDS Set Problem}

In the Partial Red-Blue Dominating Set problem, or Partial $R B D S$ for short, we are given an additional parameter $t$, the budget parameter, and the objective is to find (whether there is) a subset $S$ of $R$ with $|S| \leq s$ that dominates at least $t$ elements of $B$. The dynamic version is defined as follows.

Dynamic Partial Red-Blue Dominating Set $(P R B D S)$

Given: Two red-blue graphs $G=(R \cup B, E)$ and $G^{\prime}=\left(R \cup B, E^{\prime}\right)$; an integer $t \geq 0$; a subset $S$ of $R$ satisfying $\left|N_{G}(S)\right| \geq t$; integers $k, r \geq 0$ such that $d_{H}\left(E, E^{\prime}\right) \leq k$.

Question: Is there a subset $S^{\prime}$ of $R$ such that $d_{H}\left(S, S^{\prime}\right) \leq r$ and $\left|N_{G^{\prime}}\left(S^{\prime}\right)\right| \geq t$ ?

Similar comments as collected in Remark 1 apply here as well: we may hence assume that $E^{\prime} \subseteq E$ and that $S^{\prime} \supseteq S$.

By enforcing $t=|B|$, it is not hard to see that the previously obtained hardness results for the increment-parameter transfer (see Theorem 2). 
Corollary 4. Dynamic PRBDS is $\mathcal{W}[2]$-hard with increment-parameter $r$.

This observation lets us focus on the other two natural parameters of this problem, the edit-parameter $k$ and the target-parameter $t$.

The Partial $R B D S$ problem is known to be fixed-parameter tractable with respect to $t$. This was (equivalently) formulated in [4] in terms of Partial (Set) Cover. The currently fastest algorithm runs in randomized time $O^{*}\left(2^{t}\right)$, using polynomial space, as shown by Koutis and Williams [17] for the related Partial Dominating Set problem. To keep the paper self-contained, we are going to describe how this type of algorithm would look like for Partial $R B D S$ next. The key is a reduction to a problem that is based on the following algebraic setting. Let $X$ denote a set of variables. A monomial of degree $d$ is a product of $d$ variables from $X$, with multiplication assumed to be commutative. A monomial is called multilinear if no variable appears twice or more in the product. A polynomial $P(X)$ (over the semiring of nonnegative integers $\mathbb{N}$ ) is a linear combination of monomials with coefficients from $\mathbb{N}$. Such polynomials, along with addition and commutative multiplication, form a commutative semiring, denoted by $\mathbb{N}[X]$. The maximum degree among all monomials of $P(X)$ is called the degree of $P(X)$. An arithmetic circuit over $\mathbb{N}$ and $X$ is a directed acyclic graph. Each node of in-degree zero is an input gate, which is labeled either with an element from $\mathbb{N}$ or with a variable from $X$. The graph contains a single output node of out-degree zero. Each other node is either an addition or a multiplication gate. Arithmetic circuits are representations for polynomials from $\mathbb{N}[X]$. A polynomial $P(X) \in \mathbb{N}[X]$ contains a certain monomial if the monomial appears with a nonzero coefficient in the linear combination that constitutes $P(X)$.

Multilinear Monomial Detection $(M l D)$

Given: An arithmetic circuit $C$ representing a polynomial $P(X)$ over $\mathbb{N}$, an integer $d \geq 0$.

Question: Does $P(X)$, construed as a sum of monomials, contain a multilinear monomial of degree at most $d$ ?

Koutis and Williams [17] showed that $M l D$, parameterized by the degree parameter $d$, is fixed-parameter tractable, by providing a randomized algorithm running in time $O^{*}\left(2^{d}\right)$, using polynomial space. They used this result to prove that Partial Dominating Set can be solved in randomized $\mathcal{F P} \mathcal{T}$ time $O^{*}\left(2^{t}\right)$.

To showcase this technique, we are first explaining how to derive an analogous result for Partial $R B D S$. Let $G=(R \cup B, E)$ and $k, t \geq 0$ form an instance of Partial $R B D S$. We are going to construct a circuit $C$ for the following polynomial.

$$
P_{k}(X):=\left(\sum_{r \in R} \prod_{b \in N_{G}(r)}\left(1+z \cdot x_{b}\right)\right)^{k},
$$

where the set of variables $X$ consists of one variable $x_{b}$ for each vertex $b \in B$, as well as one additional variable $z$. Now, $P_{k}(X)$ contains a monomial of the form $z^{t} x_{b_{1}} \cdots x_{b_{t}}$ for $B^{\prime}:=\left\{b_{1}, \ldots, b_{t}\right\}$ forming a $t$-element subset of $B$ if and only if 
$B^{\prime}$ is dominated by (at most) $k$ elements from $R$. The intuition is the following: By raising the sum-of-products to the $k^{\text {th }}$ power, any monomial is formed by picking $k$ of the product-terms. As the sum ranges over all red vertices, this corresponds to selecting $\ell \leq k$ red vertices, forming $R^{\prime}=\left\{r_{1}, \ldots, r_{\ell}\right\}$. Each of these vertices from $R^{\prime}$ will dominate the whole neighborhood. However, as we need to only dominate $t$ vertices, we may select $t$ vertices (if possible) from $N_{G}\left(R^{\prime}\right)$, and moreover, each vertex $b_{i}$ from this chosen set $B^{\prime} \subseteq N_{G}\left(R^{\prime}\right)$ selects one vertex $d\left(b_{i}\right) \in R^{\prime}$ as its dominator. Consider the monomial

$$
\prod_{r_{j} \in R^{\prime}} \prod_{b \in B^{\prime}, r_{j}=d(b)} z \cdot x_{b}
$$

contained in $P_{k}(X)$. It is obviously of the required form; in particular, it is multilinear (with respect to $X \backslash\{z\}$ ) and contains $z^{t}$ but not $z^{t+1}$.

Moreover, for any other monomial $z^{t} \xi_{1} \cdots \xi_{t}$, formed in a different way, we necessarily find $1 \leq i<j \leq t$ such that $\xi_{i}=\xi_{j}$, i.e., this monomial is not multilinear. Observe that the size of $C$ is polynomial in the size of $G$, because the term of the sum-of-products need not be repeated in a circuit. Hence, one could use the randomized $M l D$-algorithm to solve Partial $R B D S$ in randomized time $O^{*}\left(2^{t}\right)$ as claimed, where the parameter $t$ becomes the degree parameter.

We show the same applies to the dynamic version, when parameterized by the edit-parameter.

Theorem 3. Dynamic Partial RBDS is fixed-parameter tractable with respect to the edit-parameter $k$.

Proof. Let $\left(G, G^{\prime}, S, k, r, t\right)$ be an instance of Dynamic Partial $R B D S$, as in the definition above. Assume $E^{\prime} \subseteq E$ and $S^{\prime} \supseteq S$. Observe that $t-\left|N_{G^{\prime}}(S)\right| \leq k$, since at most $k$ elements of $N_{G^{\prime}}(S)$ are affected by at most $k$ edge deletions. So it would be enough to dominate at most $t-\left|N_{G^{\prime}}(S)\right|$ elements of $B \backslash N_{G^{\prime}}(S)$. We can hence use the presented $\mathcal{F} \mathcal{P} \mathcal{T}$-algorithm for Partial $R B D S$, applied to the red-blue subgraph induced by $R \cup\left(B \backslash N_{G^{\prime}}(S)\right)$, with $t-\left|N_{G^{\prime}}(S)\right|(\leq k)$ as a parameter.

As we will see, the following seemingly easy generalization cannot be solved by using this algebraic approach. This proves that a rather natural variation of $M l D$ cannot be solved in $\mathcal{F} \mathcal{P} \mathcal{T}$-time. We are now turning our attention towards Partial $q-R B D S$ for arbitrary (fixed) $q \geq 1$ :

Given: A red-blue graph $G=(R \cup B, E)$, integers $k, t, s \geq 0$.

Question: Is there a subset $S \subseteq R,|S| \leq s$, and a subset $N \subseteq B$ with $|N| \geq t$ such that each element in $N$ has at least $q$ neighbors in $S$ ?

If $q$ is part of the input, we speak of Partial General $R B D S$. As a natural generalization, we again consider a need-based variation. We will return to the whole family of problems Dynamic Partial $q-R B D S$ below. First recall that in the previous section, we used the (more general) Need-Based $R B D S$ problem to address the dynamic variant of $q-R B D S$ and we showed it to be fixed-parameter tractable. Unfortunately, this is more delicate here, as we will show now. 
In the Need-Based Partial Red-Blue Dominating Set problem, we are given an integer $q$ and a function $\eta: B \longrightarrow\{0,1, \ldots q\}$, and we say that an element $v$ of $B$ is dominated, or henceforth satisfied, by a subset $D$ of $R$ if it has $\eta(v)$ neighbors in $D$. A formal definition follows.

Need-Based Partial Red-Blue Dominating Set $(N B-P R B D S)$

Given: A red-blue graph $G=(R \cup B, E)$ together with integers $s, t, q \geq 0$ and a function $\eta: B \longrightarrow\{0,1, \ldots q\}$.

Question: Does $R$ contain a subset $D$ of cardinality at most $s$ that satisfies at least $t$ elements of $B$ ?

Here, a subset $D$ of $R$ satisfies at least $t$ elements of $B$ if there is a set $B^{\prime} \subseteq B$ such that $\left|B^{\prime}\right| \geq t$ and for all $v \in B^{\prime},|N(v) \cap D| \geq \eta(v)$.

As in the previous section, we can tacitly assume that $\eta(v)>0$ for all $v \in B$, as otherwise we can easily satisfy $v$ and hence remove $v$ and decrement $t$.

It might be tempting to think that the ideas leading to $\mathcal{F} \mathcal{P} \mathcal{T}$-algorithms in Theorem 3 transfer to this case. We did try to work in this direction, but there seems to be some difficulty because different individuals have different needs, and this cannot be modeled while checking, at the same time, that at least $t$ blue vertices are dominated.

This difficulty can be backed with the following hardness result.

Theorem 4. NB-PRBDS is $\mathcal{W}[1]$-hard with respect to t and $q$ (and s) as parameters, even if the need function $\eta$ is constant.

Proof. First, observe that if $s=t=q$ and $\eta$ being the constant function $\eta=q$, $N B-P R B D S$ asks about a biclique in the bipartite graph $G=(R \cup B, E)$ with exactly $s=q$ vertices in $R$ (as $q$ is a trivial lower bound on the size of any solution) and (at least) $t=q$ vertices in $B$. Rather recently, Lin proved that it is $\mathcal{W}$ [1]-hard to find such a biclique $K_{t, t}$ in a given bipartite graph, see [20] in combination with [19, Lemma 3.1].

Corollary 5. Partial General RBDS is $\mathcal{W}[1]$-hard, parameterized with $t$, q, or s.

Observe that usually, the partial variants of domination-like problems tend to be in the class $\mathcal{F} \mathcal{P} \mathcal{T}$. To the best of our knowledge, this is the first problem variant where this question turns out to be hard. Yet, there is a catch in this assertion, which can be seen by turning to the family of problems $N B-q-P R B D S$ whose definition coincides with that of $N B-P R B D S$, apart from the fact that $q$ is no longer part of the input here. We do not have a hardness result in this case, nor do we know of algorithmic results, even not in the case when $q=2$, the case $q=1$ having been dealt with (algorithmically) above. Rather, when we look at Dynamic Partial General $R B D S$, where we have $q$ as part of the input, we can show the following result.

Corollary 6. Dynamic Partial General $R B D S$ is $\mathcal{W}[1]$-hard with respect to $t$ or $q$ as parameters. $\mathcal{W}[1]$-hardness even holds for the combined parameter $(t, q, r, k)$. 
Proof. As above, consider $t=q$. If $G^{\prime}=\left(R \cup B, E^{\prime}\right)$ (together with $\left.t=q\right)$ is an instance of $N B-P R B D S$, then we obtain $G$ by adding a $K_{q, q}$, with $s=q$. The edit-parameter is $q^{2}$, the increment-parameter would be $q$.

We are now proposing a generalization of $M l D$ that we prove to be hard when parameterized with the degree parameter. This result could be of independent interest. As it is not central to the topic of the paper, we omit its proof. Multilinear Monomial Detection with Partition ( $M l D w P$ )

Given: An arithmetic circuit $C$ representing a polynomial $P(X)$ over $\mathbb{N}$, a partition of $X$, an integer $d \geq 0$.

Question: Does $P(X)$, construed as a sum of monomials, contain a multilinear

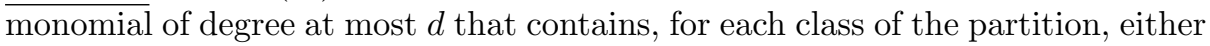
all variables in that class or no variable from that class?

Theorem 5. MlDwP, parameterized by the degree parameter, is $\mathcal{W}[1]$-hard.

Remark 4. One could try to alternatively parameterize Partial $R B D S$ by $k$ and $k^{\prime}:=|B|-t$. In fact, the problem is easily seen to be $W[2]$-hard, when parameterized by $k^{\prime}$, because the problem can then be re-formulated as follows (disregarding isolated vertices): Given some red-blue graph $G=(R \cup B, E)$ and integers $k, k^{\prime} \geq 0$, find subsets $R^{\prime} \subseteq R$ and $B^{\prime} \subseteq B$, with $\left|R^{\prime}\right| \leq k$ and $\left|B^{\prime}\right| \leq k^{\prime}$, such that $N\left(R^{\prime}\right)=B \backslash B^{\prime}$ and $N\left(B^{\prime}\right)=R \backslash R^{\prime}$. Hence, if $k=0$, the question boils down to $R B D S$ itself, with the roles of red and blue being exchanged.

Clearly, this tweak does not change the dynamic version of the problem at all, it is equivalent to Dynamic Partial $R B D S$ as studied above.

Remark 5. One could also think of changing the notion of a solution in the dynamic partial setting. This would mean the following problem.

Dynamic Partial Red-Blue Dominating Set with Blue Focus ( $R B D S B F)$

Given: Two red-blue graphs $G=(R \cup B, E)$ and $G^{\prime}=\left(R \cup B, E^{\prime}\right)$; an integer $t \geq 0$; a subset $T$ of $B$ satisfying $|T| \geq t$, dominated by $S \subseteq R$; integers $k, r \geq 0$ such that $d_{H}\left(E, E^{\prime}\right) \leq k$.

Question: Is there a subset $T^{\prime}$ of $B$, dominated by some $S^{\prime}$ with $\left|S^{\prime}\right| \leq|S|$, such that $d_{H}\left(T, T^{\prime}\right) \leq r$ and $\left|T^{\prime}\right| \geq t ?$

$\mathcal{N P}$-hardness of this variant is easily seen by starting from some $R B D S$ instance $G^{\prime}=\left(R \cup B, E^{\prime}\right)$ and a bound $s$ on the size of the red-blue dominating set. Construct $G$ from $G^{\prime}$ by selecting a subset $S \subseteq R$ with $|S|=s$ and adding $|B|$ edges (to form $E$ ) so that $S$ is a red-blue dominating set of $G$. With $t=k=|B|$ and $r=0$, we have defined all ingredients of the equivalent Dynamic $R B D S B F$ instance. This also proves that the problem is para- $\mathcal{N} \mathcal{P}$-hard for the increment-parameter $r$.

\section{Concluding Remarks}

In this paper, we undertook a multivariate analysis of Red-Blue Dominating Set. Clearly, one could also consider further parameters, for instance the loss parameter, which is the difference between $|N(S)|$ and $\left|N\left(S^{\prime}\right)\right|$. As most of our results 
are negative ones, there is surely a need for further parameterizations. Also, it would be very helpful to know if $N B-q-P R B D S$ belongs to $\mathcal{F P} \mathcal{T}$ for any fixed $q>1$, as this would also help classify the dynamic variants of $P R B S$ for fixed $q>1$. This is the most interesting open problem in that area in our opinion.

We completely neglected approximability issues so far. The more classical $D R B D S$ problem (as Set Cover reoptimization) was previously considered in $[3,21]$. Let us at least mention one positive result, concerning the natural maximization variant of Red-Blue Dominating Set, which we call BudGeTED REDBlue $k$-Dominating SET, following the tradition of the literature of these problems. Here, we search for a subset of $k$ red vertices that dominate a maximum number of blue vertices. Khuller et al. [16] considered the approximability of the Budgeted Connected $k$-Dominating Set. In this problem there is a budget $k$ on the number of vertices we can select, and the goal is to dominate as many vertices as possible with a connected set.

Theorem 6. Budgeted Red-Blue $k$-Dominating Set is polynomial-time $\frac{1}{13}(1-\varepsilon)$ approximable, for any $\varepsilon>0$.

Proof. Given any instance $G=(R \cup B, E)$ (and $k$ ) of Budgeted Red-Blue $k$-Dominating Set, we construct an instance of Budgeted Connected $k$ Dominating SeT by turning $R$ into a clique. This results in a split graph $G^{\prime}$ on which we can use the algorithm of Khuller et al. to compute an approximate solution. In any solution containing a vertex $b$ from $B, b$ can be easily replaced by any neighbor of $b$, so that we can assume that the solution $S$ is a subset of $R$. This is also true for an optimum solution. Hence, maximum solutions to the instance $(G, k)$ correspond to maximum solutions of $\left(G^{\prime}, k\right)$ and vice versa. Thus, the approximation factor of $\frac{1}{13}(1-\varepsilon)$, shown in [16] for BudGeted ConneCted $k$-Dominating SET on split graphs, also applies to our problem.

\section{References}

1. Abu-Khzam, F.N., Egan, J., Fellows, M.R., Rosamond, F.A., Shaw, P.: On the parameterized complexity of dynamic problems. Theoret. Comput. Sci. 607, 426$434(2015)$

2. Alman, J., Mnich, M., Williams, V.V.: Dynamic parameterized problems and algorithms. In: Chatzigiannakis, I., Indyk, P., Kuhn, F., Muscholl, A. (eds.) 44th International Colloquium on Automata, Languages, and Programming, ICALP. LIPIcs, vol. 80, pp. 41:1-41:16. Schloss Dagstuhl - Leibniz-Zentrum für Informatik (2017)

3. Bilò, D., Widmayer, P., Zych, A.: Reoptimization of weighted graph and covering problems. In: Bampis, E., Skutella, M. (eds.) WAOA 2008. LNCS, vol. 5426, pp. 201-213. Springer, Heidelberg (2009). https://doi.org/10.1007/978-3-540-93980$1 \_16$

4. Bläser, M.: Computing small partial coverings. Inf. Process. Lett. 85(6), 327-331 (2003)

5. Böckenhauer, H.-J., Hromkovič, J., Mömke, T., Widmayer, P.: On the hardness of reoptimization. In: Geffert, V., Karhumäki, J., Bertoni, A., Preneel, B., Návrat, P., Bieliková, M. (eds.) SOFSEM 2008. LNCS, vol. 4910, pp. 50-65. Springer, Heidelberg (2008). https://doi.org/10.1007/978-3-540-77566-9_5 
6. Bodlaender, H.L., Lokshtanov, D., Penninkx, E.: Planar capacitated dominating set is $W[1]$-hard. In: Chen, J., Fomin, F.V. (eds.) IWPEC 2009. LNCS, vol. 5917, pp. 50-60. Springer, Heidelberg (2009). https://doi.org/10.1007/978-3-642-11269$0 \_4$

7. Cai, Z., Miao, D., Li, Y.: Deletion propagation for multiple key preserving conjunctive queries: approximations and complexity. In: International Conference on Data Engineering, ICDE, pp. 506-517. IEEE (2019)

8. Cesati, M.: The turing way to parameterized complexity. J. Comput. Syst. Sci. 67(4), 654-685 (2003)

9. Cygan, M., et al.: On problems as hard as CNF-SAT. ACM Trans. Algorithms 12(3), 41:1-41:24 (2016)

10. Damaschke, P.: Parameterizations of hitting set of bundles and inverse scope. J. Comb. Optim. 29(4), 847-858 (2015)

11. Dom, M., Lokshtanov, D., Saurabh, S., Villanger, Y.: Capacitated domination and covering: a parameterized perspective. In: Grohe, M., Niedermeier, R. (eds.) IWPEC 2008. LNCS, vol. 5018, pp. 78-90. Springer, Heidelberg (2008). https:// doi.org/10.1007/978-3-540-79723-4_9

12. Downey, R.G., Egan, J., Fellows, M.R., Rosamond, F.A., Shaw, P.: Dynamic dominating set and turbo-charging greedy heuristics. Tsinghua Sci. Technol. 19(4), 329-337 (2014)

13. Fernau, H., Rodríguez-Velázquez, J.A.: A survey on alliances and related parameters in graphs. Electron. J. Graph Theory Appl. 2(1), 70-86 (2014)

14. Kao, M., Chen, H., Lee, D.: Capacitated domination: problem complexity and approximation algorithms. Algorithmica 72(1), 1-43 (2015)

15. Kearns, M.J.: Computational Complexity of Machine Learning. ACM Distinguished Dissertations. MIT Press, Cambridge (1990)

16. Khuller, S., Purohit, M., Sarpatwar, K.K.: Analyzing the optimal neighborhood: algorithms for budgeted and partial connected dominating set problems. In: Symposium on Discrete Algorithms (SODA), pp. 1702-1713. SIAM (2014)

17. Koutis, I., Williams, R.: Limits and applications of group algebras for parameterized problems. ACM Trans. Algorithms 12(3), 31:1-31:18 (2016)

18. Lenstra Jr., H.W.: Integer programming with a fixed number of variables. Math. Oper. Res. 8(4), 538-548 (1983)

19. Lin, B.: The parameterized complexity of k-biclique. In: Symposium on Discrete Algorithms (SODA), pp. 605-615. SIAM (2015)

20. Lin, B.: The parameterized complexity of the k-biclique problem. J. ACM 65(5), 34:1-34:23 (2018)

21. Mikhailyuk, V.A.: Reoptimization of set covering problems. Cybern. Syst. Anal. 46(6), 879-883 (2010)

22. Srinivasan, A.: Improved approximation guarantees for packing and covering integer programs. SIAM J. Comput. 29(2), 648-670 (1999) 